\title{
Understanding Patient Perspectives and Awareness of the Impact and Treatment of Anemia with Chronic Kidney Disease: A Patient Survey in China
}

This article was published in the following Dove Press journal: International Journal of Nephrology and Renovascular Disease

\author{
Chuan-Ming Hao' \\ Eric T Wittbrodt $\mathbb{D}^{2}$ \\ Eirini Palaka ${ }^{3}$ \\ Nicolas Guzman ${ }^{4}$ \\ Alicia Dunn ${ }^{5}$ \\ Susan Grandy (iD ${ }^{6}$
}

'Fudan University, Huashan Hospital, Shanghai, People's Republic of China;

${ }^{2}$ Biopharmaceuticals Medical Unit, AstraZeneca, Gaithersburg, MD, USA; ${ }^{3}$ Biopharmaceuticals Global Market Access, AstraZeneca, Cambridge, UK; ${ }^{4}$ Global Medicines Development, AstraZeneca, Gaithersburg, MD, USA; ${ }^{5}$ AstraZeneca Global Corporate Affairs Gaithersburg, Gaithersburg, MD, USA; ${ }^{6}$ Biopharmaceuticals Global Market Access, AstraZeneca, Gaithersburg, MD, USA
Correspondence: Chuan-Ming Hao Fudan University, Huashan Hospital, Shanghai, People's Republic of China Tel +86-2I-62480303

Email chuanminghao@fudan.edu.cn
Background: Anemia is a common complication of chronic kidney disease (CKD) that may reduce patients' health-related quality of life (HRQoL). This study explored the experience and knowledge of patients with CKD, with and without anemia, in China.

Methods: A quantitative online survey was administered to 500 consenting Chinese patient volunteers aged $\geq 18$ years with self-reported CKD, with or without anemia, between August 29, and September 17, 2018. Patients with cancer were excluded. The 27-question survey explored knowledge of anemia, HRQoL, anemia management, and interactions with healthcare providers. Results: Of 456 evaluable patients, 148 (32.5\%) reported having anemia and 262 (57.5\%) did not. Knowledge of anemia and its symptoms varied, and approximately half of all patients did not know their hemoglobin level. Patients with anemia expressed an adverse impact of anemia on HRQoL, most commonly lack of energy (65.5\%), sadness/depression (54.1\%), and feeling ill $(50.0 \%)$. The most frequently reported treatments among these patients were dietary advice (68.9\%), iron supplements (63.5\%), and oral medications (53.4\%). Although $89.2 \%$ of patients with anemia trusted their healthcare providers above other information sources, only $29.0 \%$ reported seeking information from them; this was despite $92.6 \%$ reporting wanting further information and support about managing conditions like anemia.

Conclusion: Our findings suggest that patients with CKD, both with and without anemia, would benefit from increased awareness of anemia and more in-depth discussions with healthcare providers in order to facilitate better management of CKD and optimization of treatment plans.

Keywords: anemia, chronic kidney disease, survey, patient perspectives, China

\section{Introduction}

Chronic kidney disease (CKD) is a growing health concern, both in China and the rest of the world. ${ }^{1,2}$ In a report published in 2018, there was an estimated CKD prevalence of $11.6 \%$ in the overall population of China. ${ }^{1}$ This figure is expected to increase in the future, given contributing factors such as continued changes in diet and lifestyle, an aging population ${ }^{2-4}$ and, potentially, increased disease recognition by physicians. The main cause of CKD in China is glomerulonephritis, but this may be supplanted by diabetes and hypertension in coming years as the prevalence of these conditions increases among the Chinese population. ${ }^{4}$

Anemia becomes increasingly prevalent among patients with CKD as the disease progresses, and affects almost all patients eligible for dialysis. ${ }^{5,6}$ In China, approximately half of patients with CKD not on dialysis and nearly $70 \%$ of those 
with diabetic nephropathy are affected by anemia. ${ }^{7}$ A 2015 analysis estimated the age-adjusted incidence of dialysisdependent CKD to be 122.19 per million in China. ${ }^{8}$ There are well-established serious consequences associated with anemia in CKD, including CKD progression, cardiovascular disease, and increased mortality risk. ${ }^{6,9-11}$ The presence of anemia with CKD is also associated with reduced health-related quality of life (HRQoL). ${ }^{12}$

Although treatments exist for anemia in CKD, current evidence suggests that the condition is under-recognized and undertreated in clinical practice. ${ }^{7,13,14}$ This is partly because patients with early-stage CKD often show only mild or no visible symptoms, therefore the disease is frequently not diagnosed until it has reached an advanced stage, where the risks of morbidity and mortality are significantly higher than at earlier stages. ${ }^{15,16}$ As medical care and innovative treatment options become increasingly available in China, understanding of CKD and its management are improving: ${ }^{17}$ however, published data specific to Chinese patients are scarce.

Patient insights specific to the Chinese population are important for understanding how to address the critical unmet needs of patients living with a disease or condition in China. Patients' participation in healthcare and treatment can help providers understand and consider patients' opinions and experiences, also known as the "patient voice", which may lead to improved health outcomes and increased satisfaction with healthcare. ${ }^{18}$ Patient insights can also provide information about how actively patients are involved in their own care, which is recognized as a key component of chronic disease management. ${ }^{19}$ In general, information about the experience of patients with CKD is lacking, particularly from patients without end-stage renal disease (ESRD). ${ }^{20,21}$

This study aimed to understand the experience of patients with CKD and anemia in China by means of an online survey, focusing specifically on the prevalence of anemia, knowledge about anemia, HRQoL, how the condition is treated and managed, and patients' perspectives on healthcare practitioner-patient relationships.

\section{Methods}

\section{Survey Design and Participants}

A quantitative online survey, developed in collaboration with Portland Communications (London, UK), was completed by 500 Chinese patient volunteers between August 29 and September 17, 2018. All patients invited to complete the survey had provided informed consent, were aged $\geq 18$ years, and had self-reported CKD of any stage, with or without anemia. Patients were recruited from an online community, patient associations, online support groups, and from patient referrals by the research firm, Opinion Health Ltd (London, UK), according to their standard recruitment protocol. In brief, a random sample of potential patients were invited to participate in the online survey, and response rates were monitored. Additional patients were invited in order to ensure a balanced sample composition in terms of gender, age, geographic region, and other sociodemographic characteristics. Recruitment was not subject to quotas for CKD disease stages or dialysis status.

Portland Communications worked with Opinion Health to carry out recruitment, field work, and data processing. The research was carried out in accordance with the European Pharmaceutical Market Research Association Code of Conduct, European Medicines Agency good pharmacovigilance practice guidelines, and General Data Protection Regulation guidance. All patients provided completed online consent forms to take part in the study and were financially compensated for their time.

\section{Data Collection}

The 27-question survey was developed de novo for the present study and was made available on an online platform through a weblink sent directly to the email address of each recruited participant. The survey questions were translated to both Mandarin and Cantonese by an ISOcertified translation company for the purpose of this study, with all translations subject to quality assurance checks. Prior to completing the survey questions, participants were required to complete three initial screening questions to confirm that they had CKD, and to report any comorbidities and CKD stage. In particular, patients were asked whether they had cancer or cancer-induced anemia, to avoid confounding the etiology of anemia if both CKD and cancer were present. Demographic questions were also asked regarding gender, age, and region.

The main part of the survey explored patients' knowledge of anemia, whether they had been diagnosed with anemia by a doctor (no definition of anemia was provided; patients were asked to report their hemoglobin level if they had received a blood test in the past year, but this information was not mandatory), information sources used to learn about the condition, disease management by healthcare practitioners, and relationships 
between patients and their healthcare practitioners. Some questions were asked only to patients who reported having been diagnosed with anemia, including questions about the impact of anemia on HRQoL and on the management of anemia by healthcare practitioners. The full list of survey questions is provided in the Supplementary Material. Patients were required to complete all survey questions. All information on disease status was selfreported by patients, including CKD stage, presence of anemia, and treatments received; responses were not further validated clinically. Data collected from the survey were aggregated and anonymized to protect patient confidentiality.

\section{Statistical Analysis}

The findings are reported descriptively as figures, which include $\mathrm{n}(\%)$ of patients for each response. No hypotheses were tested, and no inferential statistics were performed.

\section{Results}

Of 500 volunteers who completed the survey, 44 patients reported having cancer or cancer-induced anemia and were not included in the overall analytic cohort.

\section{Baseline Characteristics}

Baseline characteristics of the 456 survey respondents included in the overall analytic cohort are presented in Table 1. Overall, 148 patients (32.5\%) reported being diagnosed with anemia, and $262(57.5 \%)$ reported not being diagnosed with anemia. In total, 46 patients were unaware of their anemia status and were not included in either the anemia or non-anemia cohorts. Among patients with self-reported anemia, almost two-thirds were between 36 and 50 years old, and $61.5 \%$ were male. The nonanemia cohort had a greater proportion of older patients (aged 51-65 years) compared with the anemia cohort $(16.8 \%$ vs $2.7 \%)$, but patients in the non-anemia cohort were less likely to have a comorbidity (34.4\% vs $74.3 \%$ ), which was true for all of the individual conditions listed (Table 1). The most frequently reported comorbidities among patients with anemia were hypertension (36.5\%) and type 2 diabetes/high blood glucose (24.3\%).

In the overall analytic cohort, the proportions of patients with stage 1, 2, and 3 CKD were $39.0 \%, 34.4 \%$, and $16.7 \%$, respectively, with the remainder having stage 4 or $5 \mathrm{CKD}(6.6 \%)$ or being unaware of their disease stage $(3.3 \%)$. Most patients without anemia (81.3\%) had stage 1 or $2 \mathrm{CKD}$, and only $7.6 \%$ had stage $3 \mathrm{CKD}$. By contrast, the proportions of patients with self-reported anemia who had stage 1 or $2 \mathrm{CKD}$ vs stage $3 \mathrm{CKD}$ were $57.4 \%$ and $37.2 \%$, respectively.

In the anemia cohort, only $56.8 \%$ reported being treated for both CKD and anemia, and 41.2\% reported being treated just for CKD. In the non-anemia cohort, 23.7\% reported that they were not receiving any treatment for CKD, and $6.9 \%$ reported that they were being treated for both CKD and anemia (Table 1), despite having previously reported that they had not been diagnosed with anemia by a doctor.

\section{Knowledge}

Most patients with self-reported anemia (87.2\%) had been informed about the association between CKD and anemia by their doctor. However, $46.6 \%$ of these patients reported not knowing their hemoglobin $(\mathrm{Hb})$ level, and 9.5\% reported not having had a blood test in the previous year. Among non-anemic patients, 53.1\% reported being unaware of their $\mathrm{Hb}$ level, and $26.3 \%$ said that they had not undergone a blood test in the past year (Figure 1).

Among patients who knew their $\mathrm{Hb}$ level in the overall cohort $(n=125), 42$ of $73(57.5 \%)$ men reported levels below the standard anemia definition threshold of $13.0 \mathrm{~g} /$ $\mathrm{dL}$ for men, and 16 of 52 (30.8\%) women reported levels below the threshold of $12.0 \mathrm{~g} / \mathrm{dL}$ for women (Supplementary Table 1). A breakdown of $\mathrm{Hb}$ levels was not available for the anemia and non-anemia cohorts separately, so it is unclear whether this difference between men and women is driven by differences in the numbers of anemic men and women with available $\mathrm{Hb}$ levels. Within the anemia cohort, 32 of $65(49.2 \%)$ patients reported $\mathrm{Hb}$ levels under $12.0 \mathrm{~g} / \mathrm{dL}$, and eight of 54 (14.8\%) of the nonanemic patients reported $\mathrm{Hb}$ levels below this threshold.

When asked whether statements relating to the causes of anemia were true or false, understanding of the causes of anemia was, in general, slightly higher among anemic than non-anemic patients. However, a higher proportion of non-anemic patients knew that anemia could be treated (30.2\% vs $24.3 \%$; Figure 2 ). There were also some misconceptions about the causes of anemia in both cohorts, with approximately one-third of both anemic and nonanemic patients believing that anemia is caused by low blood sugar. Patients' knowledge of the symptoms of anemia appeared to vary between the cohorts with and without anemia (Figure 3). The biggest numeric difference was the belief that chest pain is a symptom of anemia, reported by $20.9 \%$ of anemic patients vs $5.3 \%$ of nonanemic patients. 
Table I Baseline Characteristics, as Self-Reported by Patients

\begin{tabular}{|c|c|c|c|}
\hline Characteristics & Overall $(N=456)^{a}$ & Anemia Cohort $(n=\mid 48)^{b}$ & Non-Anemia Cohort $(n=262)$ \\
\hline \multicolumn{4}{|l|}{ Sex, n (\%) } \\
\hline Male & $255(55.9)$ & $91(61.5)$ & $|3|(50.0)$ \\
\hline Female & $201(44.1)$ & $57(38.5)$ & $|3|(50.0)$ \\
\hline \multicolumn{4}{|l|}{ Age, years, n (\%) } \\
\hline $18-35$ & I 33 (29.2) & $50(33.8)$ & $72(27.5)$ \\
\hline $36-50$ & $265(58.1)$ & $94(63.5)$ & I 46 (55.7) \\
\hline $5 I-65$ & $58(12.7)$ & $4(2.7)$ & $44(16.8)$ \\
\hline \multicolumn{4}{|l|}{ Region, n (\%) } \\
\hline South & $109(23.9)$ & $33(22.3)$ & $68(26.0)$ \\
\hline East & $100(21.9)$ & $41(27.7)$ & $52(19.8)$ \\
\hline North & $101(22.1)$ & $34(23.0)$ & $56(21.4)$ \\
\hline Northeast & $31(6.8)$ & $7(4.7)$ & $22(8.4)$ \\
\hline Central & $58(12.7)$ & $20(13.5)$ & $30(11.5)$ \\
\hline Southwest & $49(10.7)$ & $12(8.1)$ & $28(10.7)$ \\
\hline Northwest & $8(1.8)$ & I (0.7) & $6(2.3)$ \\
\hline$\geq$ I comorbidity, n (\%) & $229(50.2)$ & $110(74.3)$ & $90(34.4)$ \\
\hline Hypertension & $132(28.9)$ & $54(36.5)$ & $56(21.4)$ \\
\hline Type 2 diabetes or high blood glucose & $56(12.3)$ & $36(24.3)$ & I5 (5.7) \\
\hline Myocardial infarction, angina, or chronic heart failure & $64(14.0)$ & $40(27.0)$ & $12(4.6)$ \\
\hline Stroke & $10(2.2)$ & $8(5.4)$ & $2(0.8)$ \\
\hline Hyperkalemia & II (2.4) & $5(3.4)$ & $5(1.9)$ \\
\hline Rheumatoid arthritis & 59 (12.9) & 31 (20.9) & $23(8.8)$ \\
\hline Other & $9(2.0)$ & $2(1.4)$ & $5(1.9)$ \\
\hline \multicolumn{4}{|l|}{ CKD stage, $\mathrm{n}(\%)$} \\
\hline 1 & $178(39.0)$ & $37(25.0)$ & $125(47.7)$ \\
\hline 2 & $157(34.4)$ & $48(32.4)$ & $88(33.6)$ \\
\hline 3 & $76(16.7)$ & $55(37.2)$ & $20(7.6)$ \\
\hline 4 & $24(5.3)$ & $4(2.7)$ & $15(5.7)$ \\
\hline 5 & $6(1.3)$ & $4(2.7)$ & I (0.4) \\
\hline Do not know & $15(3.3)$ & $0(0.0)$ & $13(5.0)$ \\
\hline \multicolumn{4}{|l|}{ 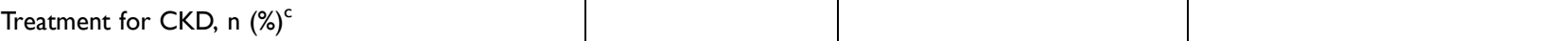 } \\
\hline Yes, for CKD but not for anemia & $275(60.3)$ & $6 I(4 I .2)$ & $182(69.5)$ \\
\hline Yes, for both CKD and anemia & $109(23.9)$ & $84(56.8)$ & $18(6.9)$ \\
\hline No & 72 (I5.8) & $3(2.0)$ & 62 (23.7) \\
\hline
\end{tabular}

Notes: All data are as reported by patients. ${ }^{\mathrm{a}}$ Of the 456 patients in the overall cohort, 46 reported being unsure or unable to remember whether their doctor had diagnosed them with anemia. ${ }^{b}$ Diagnosis of anemia by a doctor. Includes both dietary advice and pharmacological treatments.

Abbreviation: CKD, chronic kidney disease.

Knowledge of available anemia treatment options also varied considerably between the anemia and nonanemia cohorts (Figure 4). Among the anemia cohort, $85.8 \%$ of patients reported that they were made aware of available treatment options by their doctor, who either informed them proactively $(52.7 \%)$ or responded to their request for information $(33.1 \%)$. The corresponding percentages were lower $(22.5 \%$ and $24.4 \%)$ in non-anemic patients, but many said they would like to know more about anemia treatment options (30.2\%).
More patients in the anemia cohort reported feeling confident about their knowledge of the side effects of anemia treatments than in the non-anemia cohort ( $78.4 \%$ vs $38.5 \%)$.

\section{HRQoL in the Anemia Cohort}

Patients with anemia reported that anemia worsened their feelings of lethargy and illness (Figure 5). The physical symptoms most impacted by anemia were lack of energy (65.5\%) and feeling ill (50.0\%; Figure 5A). Patients also 
Hemoglobin level known

Hemoglobin level not known

No blood test in the past year

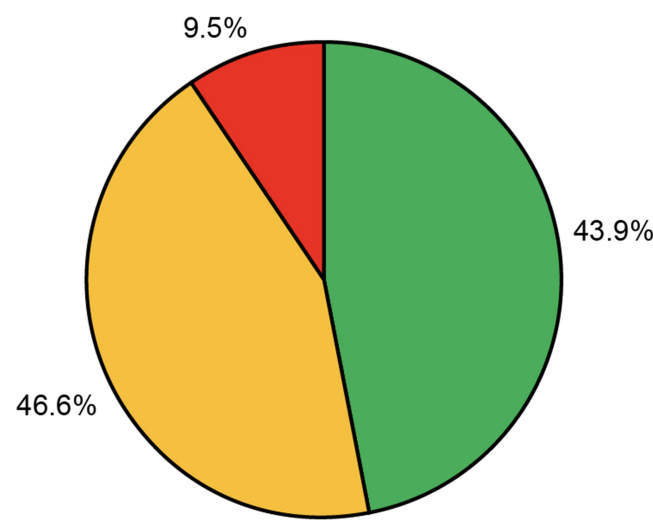

Anemia cohort $(n=148)$

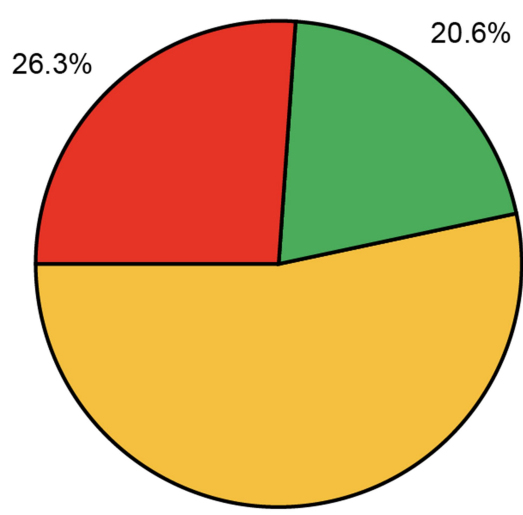

$53.1 \%$

Non-anemia cohort $(n=262)$

Figure I Knowledge of hemoglobin levels.

Notes: Charts show the percentages of patients who selected each response to the question, "If you've had a blood test in the past year, do you know what your hemoglobin level is?".

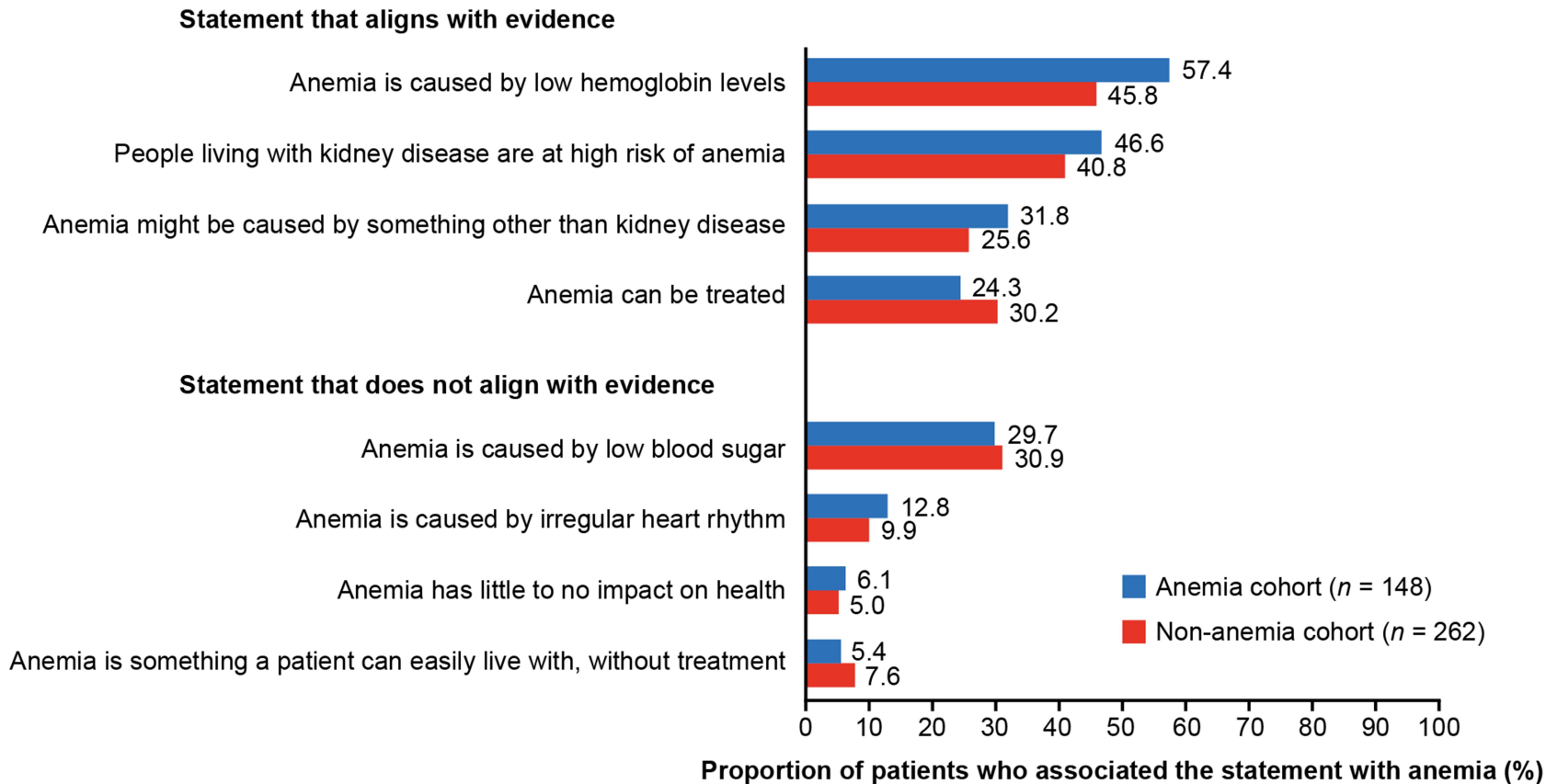

Figure 2 Beliefs about anemia and its causes.

Notes: Graph shows the percentages of patients who selected each statement in response to the question, "Which of the following statements about anemia do you think are correct?".

attributed an emotional impact to anemia, particularly on sadness/depression (54.1\%), nervousness (48.0\%), and concern about their condition worsening $(37.2 \%$; Figure 5B).

\section{Disease Management in the Anemia Cohort}

When asked about how their doctor was currently managing their anemia, patients reported receiving a variety of 


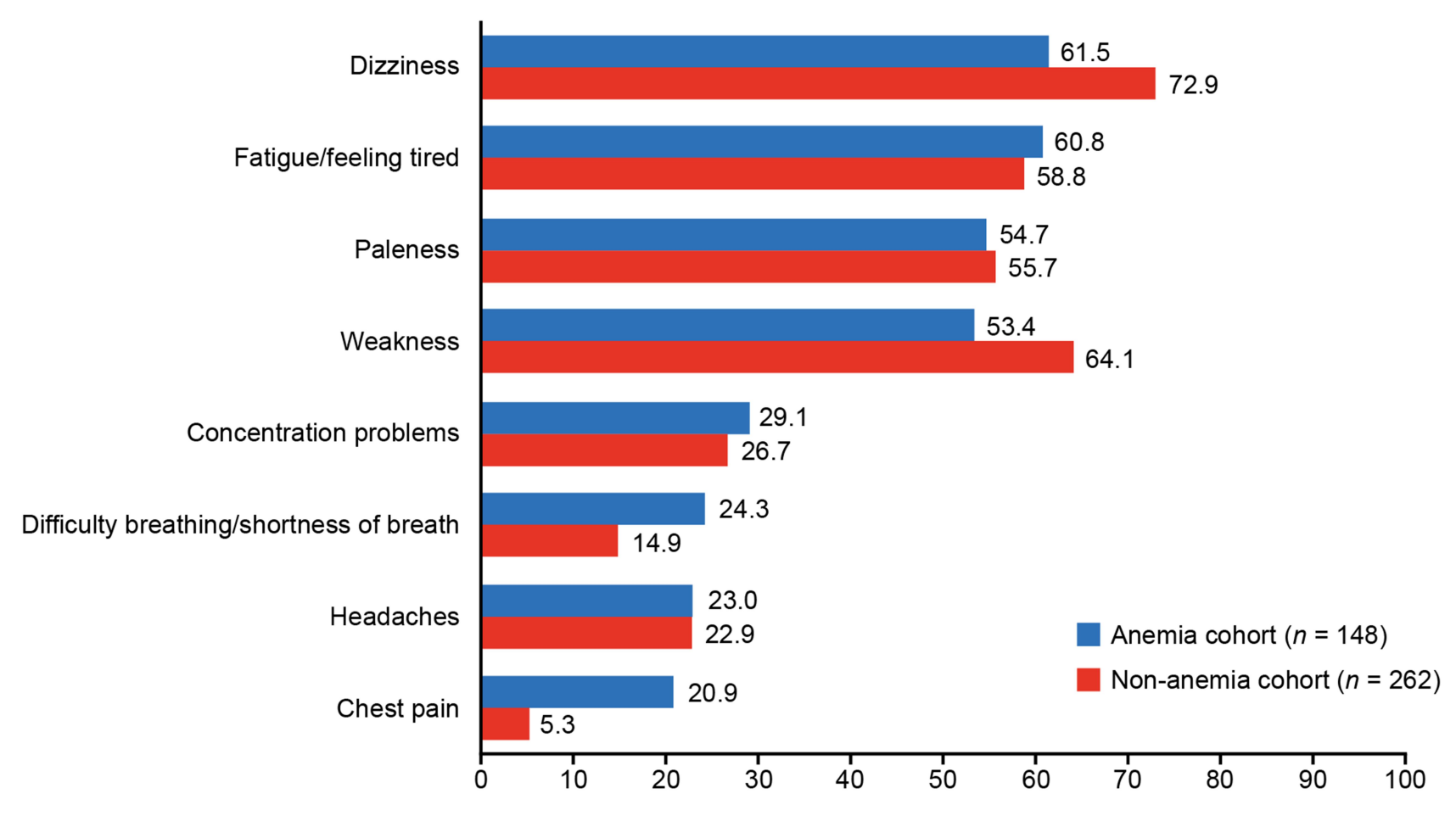

Figure 3 Beliefs about the symptoms of anemia.

Notes: Graph shows the percentages of patients who selected each statement in response to the question, "Which of the following do you think are symptoms of anemia?".

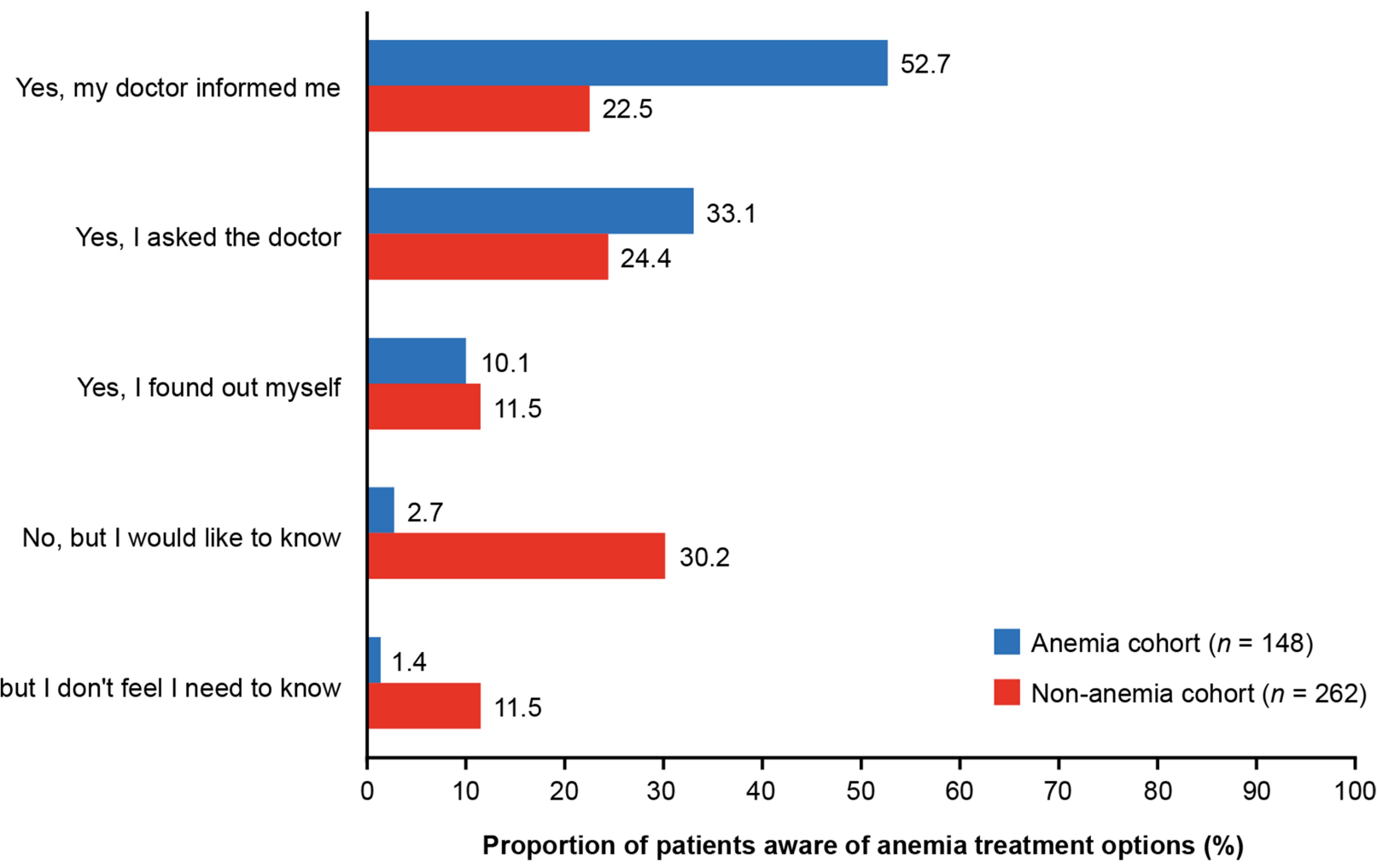

Figure 4 Knowledge of anemia treatment options.

Notes: Graph shows the percentages of patients who responded to the question, "Do you know about the different treatments that are available for managing anemia and, if so, how did you find out?". 


\section{A}

Fatigue

I have a lack of energy

I am sleeping well :-....

I am forced to spend time in bed

General malaise

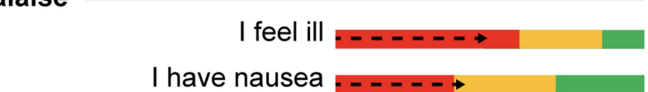

I have accepted my illness

I have pain

I am bothered by the side effects of treatment

I am content with the quality of my life
right now

\section{Daily activities}

I have trouble meeting the needs of my family

I am able to enjoy life

I am able to work (includes work at home)

My work (includes work at home) is fulfilling

I am enjoying the things I usually do for fun

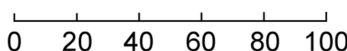

Proportion of patients $(\%)$

$\ldots$ - $\rightarrow$ Made worse by anemia
Very much/quite a bit
B

Relationships

My family has accepted my illness

I feel close to my friends

I get support from my friends

I get emotional support from my family

I am satisfied with family communication about my illness

I am satisfied with my sex life

I feel close to my partner/person who is my main support

\section{Emotions}

I feel sad and/or depressed

I feel nervous

I worry that my condition will get worse

I worry about dying

I am losing hope in the fight against

my illness

I am satisfied with how I am coping

with my illness

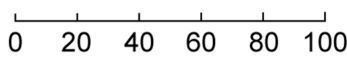

Proportion of patients (\%)

Somewhat

A little bit/not at all

Prefer not to say

Figure 5 Physical (A) and emotional (B) HRQoL (anemia cohort, $n=148$ ).

Notes: Graphs show the percentages of patients who selected each response to the question when presented with a list of statements that are commonly reported by patients with chronic conditions. Arrow length indicates the percentage of patients who stated that a particular activity or feeling was made worse by their anemia. HRQoL, health-related quality of life.

A How is your doctor managing your anemia?

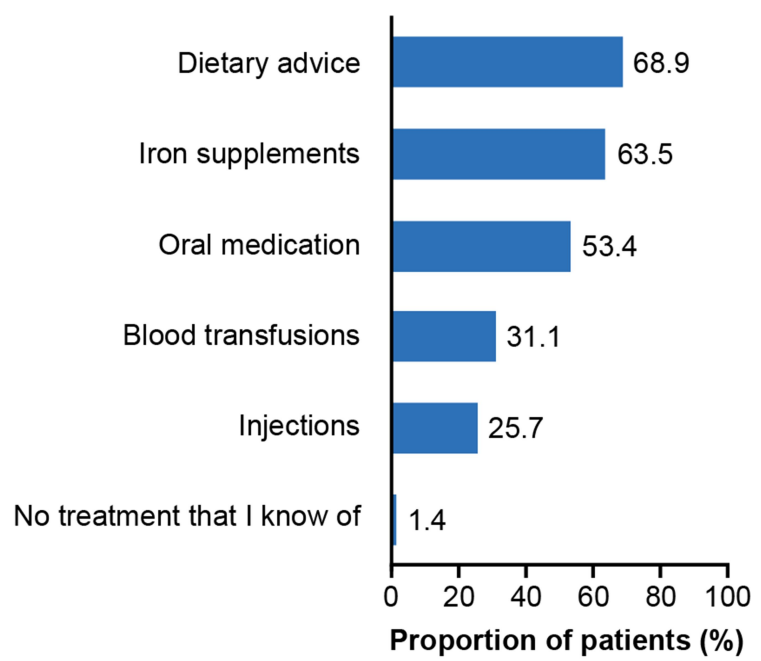

B Perception of anemia management

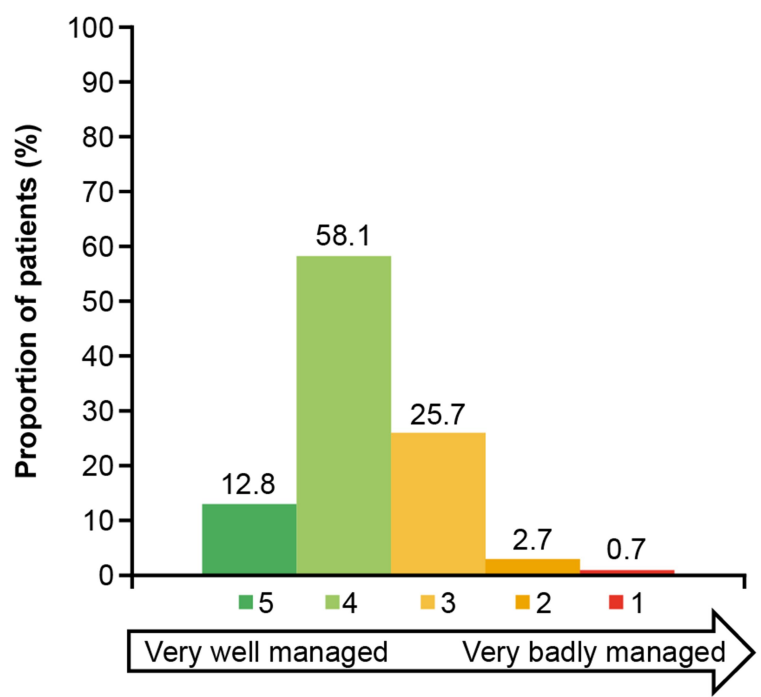

Figure 6 Patient-reported treatments received for anemia ${ }^{\mathrm{a}}(\mathbf{A})$ and patient perceptions of anemia management ${ }^{\mathrm{b}}$ (B) (anemia cohort, $\left.\mathrm{n}=148\right)$. ${ }^{\mathrm{a}}$ Percentage of patients who responded to the question, "How is your doctor managing your anemia?" bercentage of patients who selected each response to the question, "On a scale from I to 5 , how well do you think your anemia is managed, with I being 'very badly managed', 3 being 'neither badly nor well managed', and 5 being 'very well managed'?". 
A Sources used to look for information and level of trust

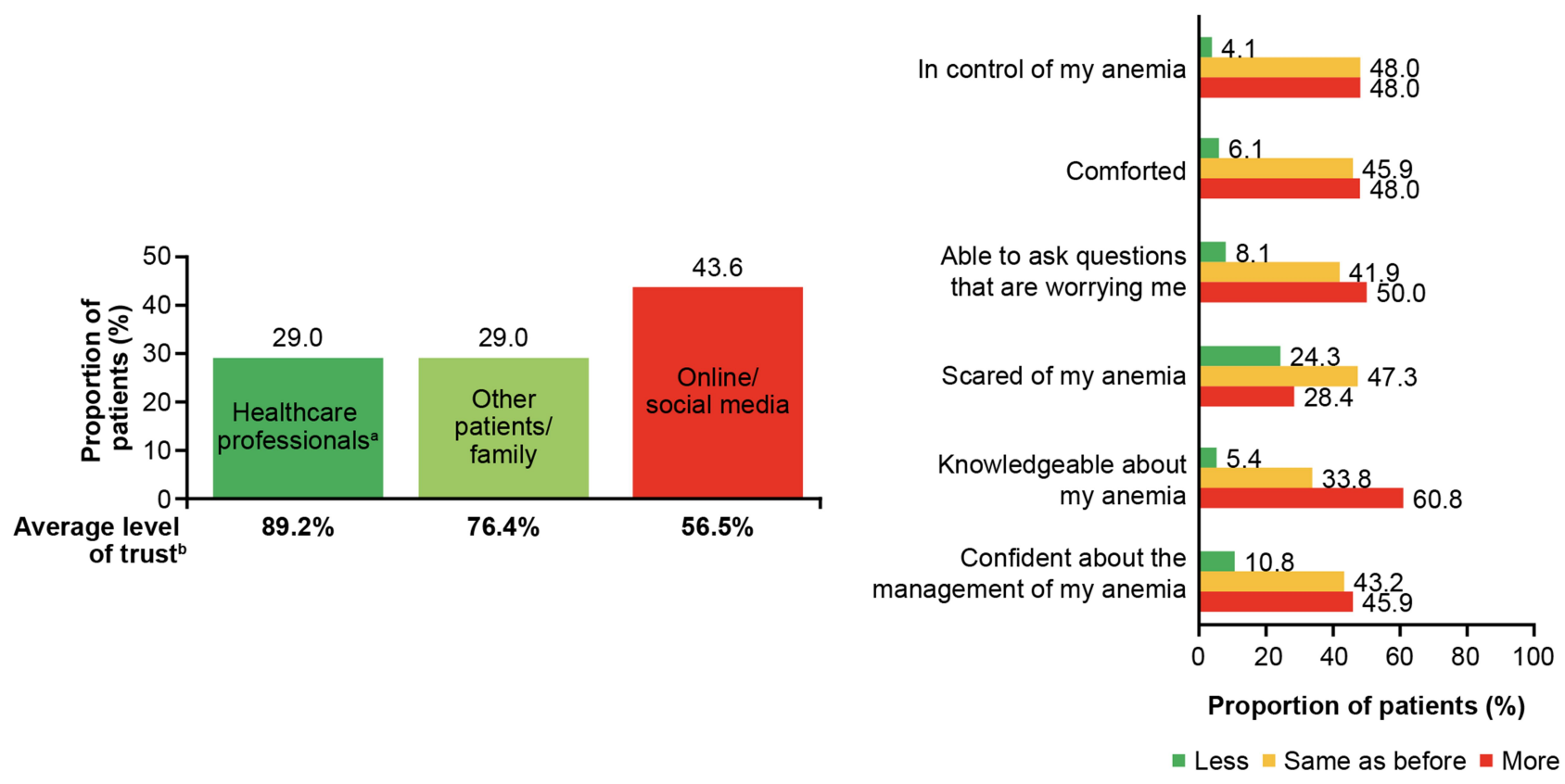

B After your discussion with your doctors about anemia, no matter how small, how do you feel?

Figure 7 Information sources and level of trust (A) and feelings after conversions with healthcare providers (B) (anemia cohort, $\mathrm{n}=\mid$ I48). ${ }^{\mathrm{a}}$ Includes nephrologist, hematologist, cardiologist, another specialist, family doctor, nurse/clinic staff, or pharmacist. ${ }^{\text {b} A v e r a g e ~ p e r c e n t a g e ~ o f ~ p a t i e n t s ~ w h o ~ r a t e d ~ t h e i r ~ l e v e l ~ o f ~ t r u s t ~ i n ~ t h e ~ i n f o r m a t i o n ~}$ sources as either a 5 or 4 on a scale of $\mathrm{I}-5$, where 5 is "completely" and I is "not at all".

therapies, most commonly dietary advice (68.9\%), iron supplements (63.5\%), and oral medications (53.4\%; Figure 6A). Almost one-third of patients (31.1\%) reported receiving blood transfusions.

Over $70.0 \%$ of patients diagnosed with anemia thought that their anemia was managed either well or very well by healthcare providers, while approximately one-quarter reported that their anemia was neither badly nor well managed. Less than $5 \%$ of patients reported that their anemia was either badly or very badly managed (Figure 6B). During regular check-ups, $65.5 \%$ of patients with anemia reported always being asked about their anemia symptoms, and $58.1 \%$ reported always having their $\mathrm{Hb}$ levels tested.

In total, $89.2 \%$ of patients with anemia trusted their healthcare providers above other information sources, despite using other sources more frequently to look for information about anemia (Figure 7A). The majority (92.6\%) of patients stated that they would like more support and information about managing their condition.

After speaking with their doctor, $45.9 \%$ of patients felt more confident about managing their anemia, and $60.8 \%$ of patients felt more knowledgeable; however, almost $30.0 \%$ of patients felt more scared about their anemia than before (Figure 7B).

\section{Discussion}

To our knowledge, the present study is the first of its kind to explore the effects of anemia in CKD on HRQoL in Chinese patients. This survey of adults with CKD in China identified varying levels of disease-specific knowledge among patients with self-reported anemia, as well as a substantial perceived burden of anemia on the HRQoL of patients participating in this survey. Patients valued interactions with their healthcare providers, indicating an opportunity to increase and strengthen the dialogue between healthcare providers and patients.

\section{Patient Characteristics, Knowledge of Anemia, and Disease Management}

Consistent with previous studies, ${ }^{14,22}$ the prevalence of comorbidities, including diabetes, hypertension, and cardiovascular conditions, was higher in the anemic cohort than in the non-anemic cohort and more anemic patients had stage 3-5 CKD. Males were disproportionately represented in the anemia cohort compared with the overall cohort, despite data suggesting that women with CKD are more at risk of anemia than men. ${ }^{22,23}$

Approximately one-third of patients with CKD surveyed reported being diagnosed with anemia, yet over half of these patients did not know their most recent $\mathrm{Hb}$ 
level and/or had not had a blood test in the previous year. In fact, one-third of anemic patients said they were never or not always asked about their anemia symptoms during regular check-ups, and over $40 \%$ did not undergo regular blood testing to check their $\mathrm{Hb}$ level. These findings appear contradictory to Kidney Disease: Improving Global Outcomes (KDIGO) guidelines for the treatment of CKD patients with anemia who are not being treated with an erythropoiesis-stimulating agent (ESA). The guidelines recommend $\mathrm{Hb}$ monitoring at least every 3 months in patients with stage 3-5 CKD not on dialysis and at least monthly in patients with stage 5 CKD on hemodialysis. ${ }^{24}$ However, it should be noted that more than half of patients in the anemia cohort had stage 1 or 2 $\mathrm{CKD}$, which may explain why their Hb levels were not being monitored this regularly. Additionally, some patients responding to the survey might have been unaware that $\mathrm{Hb}$ was being measured when undergoing blood testing; for example, if their physicians did not discuss the tests or results with them. Nevertheless, the high prevalence of anemia in the present cohort suggests a possible need for more frequent monitoring of $\mathrm{Hb}$ levels and better implementation of guidelines for anemia management.

Among patients in the overall cohort who knew their $\mathrm{Hb}$ levels, nearly one-third of women and over one-half of men were below current threshold levels for a diagnosis of anemia $(13.0 \mathrm{~g} / \mathrm{dL}$ for men and $12.0 \mathrm{~g} / \mathrm{dL}$ for women, as per KDIGO guidelines). ${ }^{24}$ In the anemia and non-anemia cohorts, $49.2 \%$ and $14.8 \%$ of patients, respectively, had $\mathrm{Hb}$ levels below $12 \mathrm{~g} / \mathrm{dL}$, suggesting that there is variation in how patients perceive and define anemia. Additionally, $56.8 \%$ of the anemia cohort reported being treated for anemia in addition to $\mathrm{CKD}$, but when asked specific questions about types of treatment $63.5 \%$ reported receiving iron supplements and $68.9 \%$ reported receiving dietary advice. This slight discrepancy suggests that some patients may have assumed a narrower definition of what constitutes anemia "treatment" than the options provided in the survey and/or perhaps did not consider "dietary advice" or over-the-counter iron to be "treatment". The interrelated nature of CKD and anemia could also have contributed to patients being unclear as to which part of their disease was being addressed by their treatment.

The proportions of patients treated for anemia with injectable agents appear higher than figures for ESA and intravenous iron use reported in a recently published analysis ${ }^{25}$ and what might be expected given the KDIGO recommended threshold for ESA treatment $(\mathrm{Hb}<10 \mathrm{~g} / \mathrm{dL}) .{ }^{24}$ The relatively high proportion of patients receiving blood transfusions is also somewhat surprising, given recommendations to avoid these when possible. ${ }^{24}$

Although the majority of patients with CKD and anemia considered their anemia to be well or very well managed, almost $30 \%$ of patients felt that their anemia was either managed badly or neither badly nor well. Over $90 \%$ said that they would like more support and information about managing their condition, and information from healthcare providers was trusted more highly than information found online, even though a high proportion of patients both with and without anemia reported looking for information about anemia on the internet. These responses emphasize the high value that patients with CKD and anemia place on their interactions with healthcare providers, suggesting an eagerness to obtain more information during consultations. Increasing dialog and disease education about anemia during consultations may improve patients' perceptions of their anemia management and might also help them self-manage their disease, which is a critical aspect of care for patients with chronic diseases. ${ }^{26}$

\section{Impact of Anemia on HRQoL}

Although knowledge of the causes and symptoms of anemia varied, it was clear that patients with self-reported anemia perceived anemia as having a considerable negative impact on their HRQoL. In particular, many patients reported having a lack of energy, feeling ill, and a negative impact of anemia on their emotional well-being, including increased feelings of sadness/depression, nervousness, and concern about their condition worsening.

These findings concur with those from studies of patients with more severe CKD than in the present study, which reported lower HRQoL among patients with anemia vs without anemia. ${ }^{6,12}$ One study reported worse HRQoL among patients with severe anemia (defined as $\mathrm{Hb}$ levels $<10 \mathrm{~g} / \mathrm{dL}$ ), with even moderate anemia (defined as $\mathrm{Hb}$ 10-12 $\mathrm{g} / \mathrm{dL}$ ) associated with poorer HRQoL scores for some measures compared with $\mathrm{Hb}>12 \mathrm{~g} / \mathrm{dL}$. ${ }^{6,12}$

A recent review of the impact of anemia on HRQoL in patients with CKD supports the notion of a correlation between $\mathrm{Hb}$ levels and HRQoL. ${ }^{27}$ The authors of this review emphasized the impact of anemia on energy/vitality and physical functioning, and noted that increases in $\mathrm{Hb}$ levels with anemia treatments may significantly improve HRQoL in patients with CKD and anemia, with the 
greatest improvements in those whose $\mathrm{Hb}$ levels are increased to the $10-12 \mathrm{~g} / \mathrm{dL}$ range. ${ }^{27}$ While the survey used in the present study was focused on patient perceptions rather than on achievement of treatment targets, such as $\mathrm{Hb}$ level, it is possible that inertia in treating anemia early in this population contributed to low HRQoL in those who were untreated.

It should be noted that the appropriate target $\mathrm{Hb}$ level in CKD remains undetermined, and KDIGO guidelines recommend individualization of therapy in patients with CKD not on dialysis who have $\mathrm{Hb}$ levels $<10 \mathrm{~g} / \mathrm{dL}$. This recommendation is due to variability in individual responses to anemia, with some patients having improvements in HRQoL at lower Hb levels. ${ }^{24}$

There are no standardized means of measuring HRQoL in patients with CKD. ${ }^{27}$ Different assessment tools used in clinical studies have included the KDQOL-36, the Kidney Disease Questionnaire, the EQ-5D, sickness impact profile, and linear analogue self-assessment. As a result, measurement of HRQoL in patients with CKD is very difficult and may fail to capture the full impact of CKD and its complications on the daily lives of affected patients. It should also be acknowledged that this study did not control for differences in other factors that might impact HRQoL, such as socioeconomic status, between the anemia and non-anemia cohorts.

To our knowledge, studies reporting on HRQoL in patients with CKD and anemia in China are limited, potentially due to a lack of robust data sets. However, some studies have investigated the impact of chronic conditions, including anemia, in Chinese patients. For example, a large population-based survey conducted in Shaanxi Province, China, showed an adverse impact of chronic diseases on HRQoL, ${ }^{28}$ and findings from the China Health and Retirement Longitudinal Study suggested that both anemia and low $\mathrm{Hb}$ levels were independent risk factors for cognitive decline. ${ }^{29}$ A small number of other studies have reported on HRQoL in Asian CKD populations. One study carried out in Japanese hemodialysis patients showed that both mental and physical component scores of the KDQOL-36 survey were both approximately 1.6 points lower in patients with $\mathrm{Hb}$ levels $<8 \mathrm{~g} / \mathrm{dL}$ compared with those who had less severe anemia (Hb levels of $11-12 \mathrm{~g} / \mathrm{dL})$. Although the difference was not statistically significant for the mental component scores, ${ }^{30}$ these findings may suggest a detrimental impact of severe anemia on physical functioning. Similarly, a Malaysian study of propensity score-matched patients with type 2 diabetes and
CKD, with or without anemia, showed that SF-36 Version 2 QoL scores were lower in anemic patients but, again, were not significantly different. ${ }^{31}$ A recent study in India also showed consistently lower EQ-5D index values for patients with diabetic kidney disease and concomitant anemia. $^{32}$

The findings from the present study concur with these findings, as the majority of patients with CKD and anemia reported an effect of anemia in worsening indicators of both physical and emotional HRQoL.

\section{Study Limitations}

This was a cross-sectional study, therefore no longitudinal data on changes in patient responses over time were collected. Although the self-reported nature of the data collected provides valuable insights direct from patients into their experience of living with CKD, the responses collected were not clinically validated and, as noted previously, there is no standardized means of assessing HRQoL in patients with CKD, which may add a level of uncertainty to the results. In particular, data on anemia status and disease stage were not confirmed using test results, treatments received were not validated using medical records, and the etiology of anemia was not assessed. For this reason, the possibility that some patients had anemia caused by other diseases cannot be excluded. The finding that 119 patients knew what their most recent $\mathrm{Hb}$ level was also adds a level of uncertainty to findings pertaining to the relationship between anemia and HRQoL outcomes.

Patients completed the survey online without the support of a healthcare provider, which might have led to differences in how study questions were interpreted. For example, some patients without anemia reported receiving treatments for anemia, possibly due to associating non-specific dietary advice with anemia. Although patients were asked about how anemia impacted their physical and emotional experience of $\mathrm{CKD}$, this was subjective, and the relative contribution of anemia and other CKD symptoms could not be determined with certainty. Additionally, although no questions were asked specifically about traditional Chinese medicines, such as herbal extracts, patients may have included these when reporting oral medication use.

The study findings should also be viewed in the context of the survey population: over $70 \%$ of patients had stage 1 or $2 \mathrm{CKD}$, and almost $90 \%$ were 50 years of age or younger. These demographics may not represent the overall population of patients with $\mathrm{CKD}$ in all regions of China. Moreover, 
the data may not provide a fully comprehensive picture of how patients with anemia are treated in China, as they may only represent a small sample of patients with CKD and anemia in China. Patients with early-stage CKD may differ in their disease knowledge and perceptions of the impact of anemia on HRQoL compared with patients with more advanced disease. Our findings may also be impacted by a sampling bias, whereby patients who agree to participate in these types of studies and who are actively involved in community activities may be more positive about their disease and/or care than average patients with CKD in China. The online nature of the survey may also mean that patients with a high level of education and/or computer literacy are more likely to participate than those who are less educated. Finally, the size of the anemia cohort in this study was relatively small and whilst our findings provide valuable information, further studies are warranted to further explore the experience of patients with CKD and anemia in China.

\section{Conclusion}

This study is among the first to provide insight into patient-specific knowledge, awareness, and perceptions of CKD and anemia in China. Findings from this study show that patients with CKD, with and without anemia, in China would benefit from increased awareness of anemia and more in-depth interactions with healthcare providers regarding anemia and its associated risks. These improvements might help patients to play a more active role in their care, to better manage their CKD and improve outcomes.

\section{Data Sharing Statement}

Data underlying the findings described in this manuscript may be obtained in accordance with AstraZeneca's data sharing policy described at: https://astrazenecagroup-dt. pharmacm.com/DT/Home.

\section{Acknowledgments}

We thank the patients who participated in this study for their contributions to progressing the understanding of chronic kidney disease and its impact on patients' lives. Medical writing support was provided by Lucy Ambrose, DPhil (Oxon), and editorial support was provided by Bethany King, both of Core Medica, London, UK, supported by AstraZeneca according to Good Publication Practice guidelines (Link).

\section{Author Contributions}

Study design and data collection: C.M. Hao, A. Dunn, E. Palaka, E.T. Wittbrodt, S. Grandy. All authors contributed to data analysis, drafting or revising the article, have agreed on the journal to which the article will be submitted, gave final approval of the version to be published, and agree to be accountable for all aspects of the work.

\section{Funding}

This study was funded by AstraZeneca. The sponsor was involved in the study design, collection, analysis, and interpretation of data, as well as data checking of information provided in the manuscript. However, ultimate responsibility for opinions, conclusions and data interpretation lies with the authors.

\section{Disclosure}

C.M. Hao has received honoraria from AstraZeneca and FibroGen. E.T. Wittbrodt, N. Guzman, A. Dunn, and S. Grandy are employees and stockholders of AstraZeneca. E. Palaka is a former employee and stockholder of AstraZeneca. The authors report no other conflicts of interest related to this work.

\section{References}

1. Wang F, He K, Wang J, et al. Prevalence and risk factors for CKD: a comparison between the adult populations in China and the United States. Kidney Int Rep. 2018;3(5):1135-1143. doi:10.1016/j. ekir.2018.05.011

2. Peng Z, Wang J, Yuan Q, et al. Clinical features and CKD-related quality of life in patients with CKD G3a and CKD G3b in China: results from the Chinese Cohort Study of Chronic Kidney Disease (C-STRIDE). BMC Nephrol. 2017;18(1):311. doi:10.1186/s12882017-0725-0

3. Zhang L, Chapter LZ. 4 - Chronic kidney disease in China. In: GarciaGarcia G, Agodoa L, Norris K, editors. Chronic Kidney Dis Disadv Pop. Cambridge, MA: Academic Press; 2017:27-31.

4. Wang J, Zhang L, Tang SC-W, et al. Disease burden and challenges of chronic kidney disease in North and East Asia. Kidney Int. 2018;94 (1):22-25. doi:10.1016/j.kint.2017.12.022

5. McClellan W, Aronoff SL, Bolton WK, et al. The prevalence of anemia in patients with chronic kidney disease. Curr Med Res Opin. 2004;20(9):1501-1510. doi:10.1185/030079904X2763

6. Hoshino J, Muenz D, Zee J, et al. Associations of hemoglobin levels with health-related quality of life, physical activity, and clinical outcomes in persons with stage 3-5 nondialysis CKD. J Renal Nutr. 2020; S1051-S2276(19):30414-30415.

7. Li Y, Shi H, Wang W-M, et al. Prevalence, awareness, and treatment of anemia in Chinese patients with nondialysis chronic kidney disease: first multicenter, cross-sectional study. Medicine. 2016;95(24):e3872. doi:10.1097/MD.0000000000003872

8. Zhang L, Zhao M-H, Zuo L, et al. China Kidney Disease Network (CK-NET) 2015 Annual Data Report. Kidney Int Suppl. 2019;9(1):e1e81. doi:10.1016/j.kisu.2018.11.001 
9. Awan AA, Walther CP, Richardson PA, Shah M, Winkelmayer WC, Navaneethan SD. Prevalence, correlates and outcomes of absolute and functional iron deficiency anemia in nondialysis-dependent chronic kidney disease. Nephrol Dial Transplant. 2019. doi:10.1093/ndt/gfz1192

10. Toft G, Heide-Jorgensen U, van Haalen H, et al. Anemia and clinical outcomes in patients with non-dialysis dependent or dialysis dependent severe chronic kidney disease: a Danish population-based study. J Nephrol. 2020;33(1):147-156. doi:10.1007/s40620-019-00652-9

11. Babitt JL, Lin HY. Mechanisms of anemia in CKD. J Am Soc Nephrol. 2012;23(10):1631-1634. doi:10.1681/ASN.2011111078

12. Eriksson D, Goldsmith D, Teitsson S, Jackson J, van Nooten F. Cross-sectional survey in CKD patients across Europe describing the association between quality of life and anaemia. BMC Nephrol. 2016;17(1):97. doi:10.1186/s12882-016-0312-9

13. Stack AG, Alghali A, Li X, et al. Quality of care and practice patterns in anaemia management at specialist kidney clinics in Ireland: a national study. Clin Kidney J. 2018;11(1):99-107. doi:10.1093/ $\mathrm{ckj} / \mathrm{sfx} 060$

14. Stauffer ME, Fan T, Moura IC. Prevalence of anemia in chronic kidney disease in the United States. PLoS One. 2014;9(1):e84943. doi:10.1371/journal.pone.0084943

15. Bidin MZ, Shah AM, Stanslas J, Seong CLT. Blood and urine biomarkers in chronic kidney disease: an update. Clin Chim Acta. 2019;495:239-250. doi:10.1016/j.cca.2019.04.069

16. Kopyt NP. Chronic kidney disease: the new silent killer. $J \mathrm{Am}$ Osteopathic Assoc. 2006;106(3):133-136.

17. Liu Z-H. Nephrology in China. Nat Rev Nephrol. 2013;9(9):523-528. doi:10.1038/nrneph.2013.146

18. Vahdat S, Hamzehgardeshi L, Hessam S, Hamzehgardeshi Z. Patient involvement in health care decision making: a review. Iran Red Crescent Med J. 2014;16(1):e12454. doi:10.5812/ircmj.12454

19. Longtin Y, Sax H, Leape LL, Sheridan SE, Donaldson L, Pittet D. Patient participation: current knowledge and applicability to patient safety. Mayo Clin Proc. 2010;85(1):53-62. doi:10.4065/ mcp. 2009.0248

20. Teasdale EJ, Leydon G, Fraser S, Roderick P, Taal MW, TonkinCrine S. Patients' Experiences After CKD Diagnosis: a Metaethnographic Study and Systematic Review. Am J Kidney Dis. 2017;70(5):656-665. doi:10.1053/j.ajkd.2017.05.019

21. Clarke AL, Yates T, Smith AC, Chilcot J. Patient's perceptions of chronic kidney disease and their association with psychosocial and clinical outcomes: a narrative review. Clin Kidney J. 2016;9 (3):494-502. doi:10.1093/ckj/sfw014
22. Wong MMY, Tu C, Li Y, et al. Anemia and iron deficiency among chronic kidney disease Stages 3-5ND patients in the Chronic Kidney Disease Outcomes and Practice Patterns Study: often unmeasured, variably treated. Clin Kidney J. 2020;13(4):613-624. doi:10.1093/ ckj/sfz091

23. Zuo L, Wang M, Hou F, et al. Anemia Management in the China Dialysis Outcomes and Practice Patterns Study. Blood Purification. 2016;42(1):33-43. doi:10.1159/000442741

24. Kidney Disease: Improving Global Outcomes (KDIGO) Anemia Work Group. KDIGO Clinical Practice Guideline for Anemia in Chronic Kidney Disease. Kidney Int Suppl. 2012;2(4):279-335.

25. Park H, Liu X, Henry L, Harman J, Ross EA. Trends in anemia care in non-dialysis-dependent chronic kidney disease (CKD) patients in the United States (2006-2015). BMC Nephrol. 2018;19(1):318. doi:10.1186/s12882-018-1119-7

26. Huygens MWJ, Vermeulen J, Swinkels ICS, Friele RD, van Schayck OCP, de Witte LP. Expectations and needs of patients with a chronic disease toward self-management and eHealth for self-management purposes. BMC Health Serv Res. 2016;16(1):232. doi:10.1186/s12913-016-1484-5

27. Finkelstein FO, Finkelstein SH. The impact of anemia treatment on health-related quality of life in patients with chronic kidney disease in the contemporary era. Adv Chronic Kidney Dis. 2019;26(4):250-252. doi:10.1053/j.ackd.2019.04.003

28. Tan Z, Liang Y, Liu S, et al. Health-related quality of life as measured with EQ-5D among populations with and without specific chronic conditions: a population-based survey in Shaanxi Province, China. PLoS One. 2013;8(7):e65958. doi:10.1371/journal.pone.0065958

29. Qin T, Yan M, Fu Z, et al. Association between anemia and cognitive decline among Chinese middle-aged and elderly: evidence from the China health and retirement longitudinal study. BMC Geriatrics. 2019;19(1):305. doi:10.1186/s12877-019-1308-7

30. Akizawa T, Pisoni RL, Akiba T, et al. Japanese haemodialysis anaemia management practices and outcomes (1999-2006): results from the DOPPS. Nephrol Dial Transplant. 2008;23(11):3643-3653. doi:10.1093/ndt/gfn346

31. Azmi S, Goh A, Muhammad NA, Tohid H, Rashid MRA. The cost and quality of life of Malaysian type 2 diabetes mellitus patients with chronic kidney disease and anemia. Value Health Regional Issues. 2018;15:42-49. doi:10.1016/j.vhri.2017.06.002

32. Hussain S, Habib A, Najmi AK. Anemia prevalence and its impact on health-related quality of life in Indian diabetic kidney disease patients: evidence from a cross-sectional study. J Evid Based Med. 2019;12(4):243-252. doi:10.1111/jebm.12367
The International Journal of Nephrology and Renovascular Disease is an international, peer-reviewed open-access journal focusing on the pathophysiology of the kidney and vascular supply. Epidemiology, screening, diagnosis, and treatment interventions are covered as well as basic science, biochemical and immunological studies. The manuscript management system is completely online and includes a very quick and fair peer-review system, which is all easy to use. Visit http://www.dovepress.com/testimonials.php to read real quotes from published authors. 